\title{
A new framework for video data retrieval using hierarchical clustering technique
}

\author{
D.Saravanan, J.Surendiran
}

\begin{abstract}
The amount of information produced every year is rapidly growing due to many factor among all media, video is a particular media embedding visual, motion, audio and textual information. Given this huge amount of information we need general framework for video data mining to be applied to the raw videos (surveillance videos, news reading, Person reading books in library etc.).We introduce new techniques which are essential to process the video files. The first step of our frame work for mining raw video data in grouping input frames to a set of basic units which are relevant to the structure of the video. The second step is charactering the unit to cluster into similar groups, to detect interesting patterns. To do this we extract some features (object, colors etc.)From the unit. A histogram based color descriptors also introduced to reliably capture and represent the color properties of multiple images. The preliminary experimental studies indicate that the proposed framework is promising.

KEYWORDS: $\quad$ Data Mining, Video Segmentations, Video Data Mining, Clustering, Histogram, Video Data Clustering.
\end{abstract}

\section{INTRODUCTION:}

Data mining, which is defined as the process $\mathrm{f}$ extracting previously unknown knowledge, and detecting interesting patterns from massive set of data, has been a very active research. As result, several commercial product and research prototypes are even available nowadays. Multimedia data mining has been performed for different types of Multimedia data: image, audio and video [1]. An example of image data mining is CONQUEST [2] system that combines satellite data with geophysical data to discover patterns in global climate change. The SKICAT systems[3] intergrades techniques for image processing and data classification in order to identify 'sky objects' captured in a very large satellite picture set. The MULTIMEDIAMINER [4] project has constructed many image understanding, indexing and mining techniques in digits media. Digital multimedia differs from previous forms of combined media in that bits representing, text, images, audios, and videos can be treated as data by computer programs. One facet of these diverse data in terms of underlying models and formats is that they are synchronized and integrated hence, can be treated as integrated data records. The collection o such integral data records constitute multimedia data set. The challenge of extracting meaningful patterns form such data sets has lead to research and development in the area of multimedia data mining.
This is a challenging field due to the non-structured nature of multimedia data. Such ubiquitous data is required in many application such an $\mathrm{s}$ financial, medical, advertising and command, control, communication and intelligence. In Video Data mining, data bases are widespread and multimedia data sets are extremely large, there are tools fro managing and searching within such collections, but the need for tolls to extract hidden and useful knowledge embedded with in data is becoming critical for much decision - making applications.

\section{VIDEO DATA MINING:}

Here we define video data mining as finding correlations and patterns previously unknown, the current status of video data mining remains mainly at the pre-processing stage, in which the preliminary issues such as video clustering, and video classification are being examined and studied for the actual mining.

\subsection{Requirements of a Video Mining System: \\ The following requirements for a video mining} system [5].

1. It should be unsupervised.

2. It should not have any assumption about the data.

3. It should uncover interesting events.

Note that requirements 2 and 3 are somewhat contradictory, since the notion of 'interesting' is subjective, and highly dependent on knowledge of the content. Our aim is to find out how few assumptions can make about the content without detecting events that are too general to be meaningful. This would help us understand the content, and help set up framework of systematic use of domain knowledge

\section{Video Classification:}

Classification is a way to categorize or sassing class labels to a pattern set under the supervision. Decision boundaries are generated to discriminate between patterns belonging to different classes. The data set is initially partitioned into segments and the classifier is trained on the former. A framework to enable semantic video classification and indexing in a specific video domain was proposed. A method for classification of different kinds of videos that uses the output of a concise video summarization technique that forms a list of key frames was present. 


\section{A new framework for video data retrieval using hierarchical clustering technique}

\section{Video Clustering}

Clustering is useful technique for the discovery of some knowledge from data set. It maps a data item into one of several clusters, where clusters are natural grouping for data items based on similarity metrics or probab8ility density models. Clustering consists of partitioning data into homogeneous granules or groups, based on some objective function that maximizes the inter-cluster distance. Video clustering has some differences with convential clustering algorithms, As mentioned earlier, due to the unstructured nature do video data, preprocessing of video data by using image processing $\mathrm{g}$ or computer vision techniques is required to get structured format features, Another difference in video clustering is that the time factor should be considered while the video data is processed.

Traditional clustering algorithms can be categorized into two main types: Partitioned and hierarchical clustering [6].Hierarchical algorithms determine all cluster at once. Hierarchical algorithms can be agglomerative ("bottom up") or divisive ("top down"). Agglomerative algorithms begin with each element as a separate cluster and merge them into successively larger clusters. Divisive algorithms begin with the whole set and proceed to divide it into successively smaller clusters. Two-way clustering, coclustering or bi clustering are clustering methods where not only the objects are clustered but also the features of the objects, i.e. if the data is represented in a data matrix, the rows and columns are clustered simultaneously.

\section{Pattern Finding:}

A general framework for video data mining was proposed to address the issue of how to extract previously unknown knowledge and detect interesting patterns. In the work, how-to segment the incoming raw video stream into meaniningful pieces, and how to extract and represent some feature (i.e. motion, object difference, text) for characterizing the segmented pieces. Then, the motion in a video sequence is expressed as an accumulation o $\mathrm{f}$ quantized pixel differences among all frames in the video segment's video data base management framework and its strategies for vide content structure and events its strategies for video contentstructure and events mining were introduced. Fig 1 shows the proposed framework.

STAGE 1: The first stage of the frame work for mining raw video data is grouping input frames to a set of basic units which re relevant to the structure of the video. This is one of the most important takes since it is the first step to construct the building blocks of the video data base.

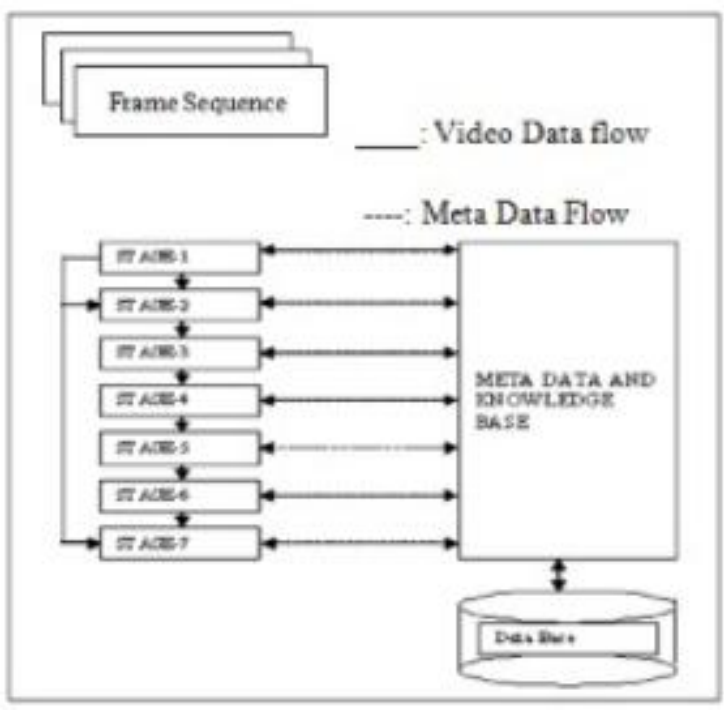

Fig 1: Proposed Framework for Video Data Mining

STAGE 2: The SECOND STAGE extracts the features such as motion, object colors etc to characterize these segments. For our frame work we consider three features (motion, object, and colors) extracted from each segments. To extract motion we make calculations using quantized pixel difference among all frames in segment.

STAGE 3: In stage THREE unwanted segments are removed by using clustering technique in clustering we employ multilevel hierarchical clustering approach to group segments with similar categories in the top level and similar motion in the bottom level.

STAGE 4: The NEXT STAGE is actual mining of raw video sequence using CLUSTERING technique. STAGE 5: In this stage the actual mining of raw video sequences processed in the above stages, and video data compression for storage of these raw videos.

STAGE 6: In the stage 6 and 7 we going to create a indexing for the video file. The meta data \& Knowledge Base in the fig a module to store the results from each stage and provide the necessary information to the stage.

\section{IMAGE MINING}

There are two major issues that will affect the image data mining process. One is the notion of similarity matching and the other is the generality of the application area. For a specific application area[7], associated domain knowledge can be used to improve the data mining task. Since data mining relies on the underlying querying capability of the CBIR system, which is based on similarity matching, user interaction will be necessary to refine the data mining process. With image mining we will consider the four broad problem areas associated with data mining:

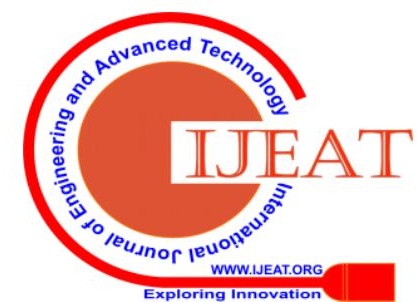


Finding associations, Classification, Sequential pattern and Time series pattern. With all these, the essential component in image mining is identifying similar objects in different images

\subsection{Image Mining Algorithm Steps:}

The algorithms needed to perform the mining of associations within the context of image. The four major image mining steps are as fallows:

1. Feature Extracting: Segment images into regions identifiable by region descriptors (blobs) ideally one blob represents one object.

2. Object identification and record creation: Compare objects in one image to objects in every other image. Label each object with an id. We call this step the preprocessing algorithm.

3. Create auxiliary images: Generate image with identified objects to interpret the association rules.

4. Apply data mining algorithm to produce object.

\section{HISTOGRAM SEARCH}

Histogram search algorithms [8],[9] characterize an image by its color distribution or histogram. A histogram is nothing but a graph that represents all the colors and the level of their occurrence in an image irrespective of the type of the image. Few basic properties about an image can be obtained from using a Histogram. It can be used to set a threshold for screening the images. The shape and the concentration of the colors in the histogram will be the same for similar objects even though they are of different colors.

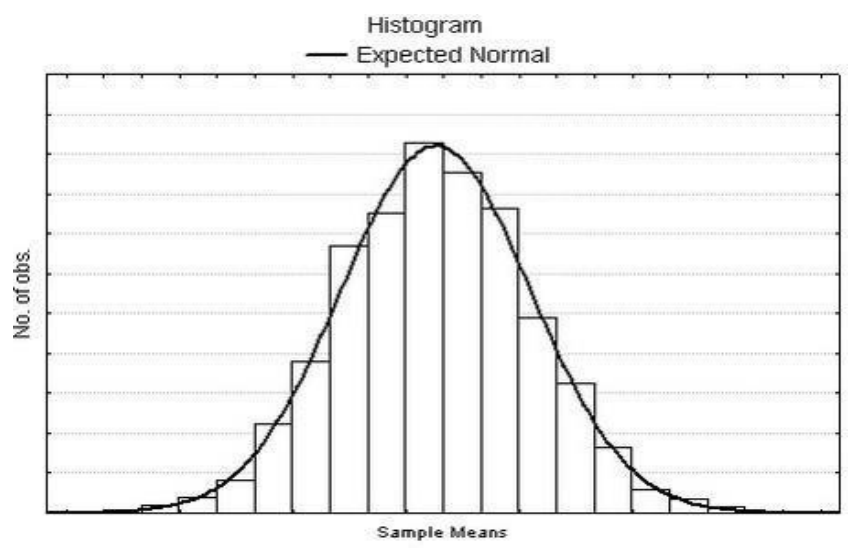

Fig 2. By using Histogram Data Analysis

\section{EXPERIMENTAL RESULTS}

Different experiments were performed, one for assessing computational performance, and one assessing robustness with respect to validate the methods we have described, we implemented the components of the video frame based retrieval system and tested with a general purpose image database including about 100 videos[10]. The table given below shows the time taken for Splitting Number of frames from the image database.

\begin{tabular}{|c|c|}
\hline Number Of Frames Spitted & Search Time \\
\hline 100 & 2 Seconds \\
\hline 1000 & 5 Seconds \\
\hline 10000 & 14 Seconds \\
\hline
\end{tabular}

Tab 1: Experimental Result of Spitted Frames

\begin{tabular}{|l|l|}
\hline \multicolumn{1}{|c|}{ Number Of Frames } & \multicolumn{1}{c|}{ Videos } \\
\hline 13116 & Cartoon \\
\hline 27956 & Tennis \\
\hline 71379 & News \\
\hline 13254 & Movie Song \\
\hline
\end{tabular}

Tab 2: Avg Search Time for Particular Frame

Mathematical operations are finally conducted in the following manner:

First a codebook of frame - tregions is created on a set of training videos.

The co-occurrence matrix is constructed:

Let $\mathrm{A}$ of size $\mathrm{M}$ by $\mathrm{N}$ be the co-occurrence matrix of $\mathrm{M}$ centroids (defining a codebook) into $\mathrm{N}$ - key frames (representing the video database).its value at cell (i, j) corresponding to the $n 8$ mber of times the region I appears in the frame $\mathrm{j}$ [11].

Next, it is analyzed through LSA:

The SVD decomposition gives $A=U_{S V}{ }^{i}$ where $\mathbf{U U}^{\mathbf{i}}=\mathbf{V} \mathbf{V}^{\mathbf{i}}$ $=\mathrm{I}, \mathrm{L}=\min (\mathrm{M}, \mathrm{N}) \mathrm{S} \approx \operatorname{diag}(\sigma 1, \ldots \ldots . . \sigma \mathrm{L}), \sigma 1 \geq \sigma 2 \geq \ldots . . \sigma \mathrm{L}$ Then $\mathrm{A}$ is approximated by truncating $\mathrm{U}$ and $\mathrm{V}$ matrices to keep $\mathrm{k}$ factors in $\mathrm{S}$ corresponding to the highest singular values.

$\hat{\mathbf{A}}=\mathbf{U k} \mathrm{Sk} \mathrm{Vk}^{\mathbf{t}}$ with $\mathbf{S k}=\operatorname{diag}(\sigma 1, \ldots \ldots . . \sigma k)$ Finally, indexing of a context of a noted $\mathrm{c}(\mathrm{j})$ and a new context $\mathrm{q}$ is realized as follows:

$P C(j)=$ row $j$ of $V S$

$$
\mathbf{U p}=\mathbf{q}^{\mathbf{t}} \mathbf{U} \mathbf{k}
$$

And to retrieve the context $\mathrm{q}$ in a database containing indexed contexts JPL, the cosine measure $\mathrm{mc}$ is used to compare elements.

$\mathrm{m}_{\mathrm{c}}\left(\mathrm{Pj}_{\mathrm{j}} \mathrm{q}\right)=\mathrm{Pq}_{\mathrm{q}} \cdot \mathrm{Pj}_{\mathrm{j}} /\left\|\mathrm{Pq}_{\mathrm{q}}\right\| .\left\|\mathrm{Pj}_{\mathrm{j}}\right\|$ the most similar elements to the query are those with the highest value of $\mathrm{m}_{\mathrm{C}}$.

The number of singular values kept for the projection drives the LSA performance. On one hand too many factors are kept, the noise will remain and the detection of synonyms and polysemy of visual terms will fail. On the other hand if too few factors are kept, important information will be lost degrading performances. Unfortunately no solution has yet been found and only experiments allow finding the appropriate factor number.

In the particular situation of video content, many features can be extracted 


\section{A new framework for video data retrieval using hierarchical clustering technique}

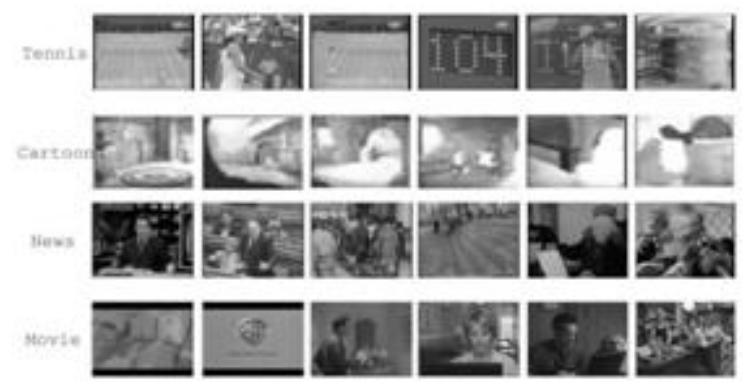

Fig.3 the model Input Frame

\section{CONCLUSION:}

In this paper, we propose a general framework for video data mining to perform the fundamental tasks which are temporal segmentation of video sequences, feature extraction, and clustering of segments. Although the experimental data showing that the proposed framework is performing the fundamental tasks effectively and efficiently.

\section{REFERENCES:}

1. Jung Hwan Oh, Babitha Bandi. Multimedia data mining framework for raw video sequences. In Proc. Of International Wrokshop on Multimedia Data Mining (with ACM SIGKDD 2002), Pages 01-10, July 2002.

2. P.Stolorz, H.Nakamura, E.Mersrobian, R.Muntz, E.Shek, J Santos, J Yi, $\mathrm{K}$ Ng, S.Chien, C.Mechoso, and J.Farrara. Fast spatial-temporal data mining of large geophysical data sets. In Proc. Of Int'l Conf. on KDD ,pages 300-305, 1995.

3. D.Saravanan, "Video data image retrieval using - BRICH", World journal of Engineering, Vol.14, Issuu 4, Pages 318-323, Aug 2017.

4. D.Saravanan," Image frame mining Using indexing technique" Data Engineering and Intelligent Computing , SPRINGER Book series, Chapter 12, Pages 127-137, ISBN:978-981-10-3223-3,July 2017.

5. U .Fayyad, S.Djorgovski, and N.Weir, Automating the analysis and cataloging of sky serverys. Advances in Knowledge Discovery with Data Mining, Pages 471-493, 1993.

6. Z .N Li, O.R.Zaiane, and Z.Tauber. Illumination invariance and object model in Content-based image and video retrieval. Journal of Visual Communication and Image Representation, 1998.

7. Ajay Divakaran, Dadir A.Peker, Shih-Fu-Change, Regunathan Radhakrishanan, Lexing Xie. Video Mining: Pattern Discovery versus pattern Recognition. In Proc. Of Int'l Conference on ICICS ,2006.

8. Ying Liu, Dengsheng Zhang, Guojun Lu, Wei-Ying Ma, " A survey of Content -based image retrieval with high-level semantics", The journal of Pattern Recognition Society, Vol 40,

9. ,pp 262-282,2007.

10. R. Benmokhtar and B. Huet,"Neural network combining classifier based on Dempster-Shafer theory for semantic indexing in video content," International MultiMedia Modeling Conference, vol. 4351, pp. 196-205, Singapore, 2007.

11. D.Saravanan" Effective Video Data Retrieval Using Image Key frame selection", Advances in Intelligent Systems and computing, Pages 145-155,jan-2017.

12. D.Saravanan, "Clustering of video in formations using BRICH Methodology" Pak journal of biotechnology, Vol 14(Special Issue-II2017), Pages 377-380, Dec- 2017

13. Steyvers, M., Smyth, P., Rosen-Zvi, M., \& Griffiths, T.(2004). Probabilistic Author-Topic Models for Information Discovery. The Tenth ACM SIGKDD International Conference on Knowledge Discovery and Data Mining. Seattle, Washington. 113-141, 2000.

14. D.Saravanan, Dr.S.Srininvasan Video Data mining pattern discovered using statistical modeling approach. In the proc. Of Int'l Confrence on Scinece and Applications ICISA 2010.,pp 353-355.

15. Nevenka Dimitrova, Hong-Jiang Zhang, Behzad Shahraray, Ibrahim Sezan, Thomas Huang, and Avideh Zakhor, "Applications of videocontent analysis and retrieval," IEEE Multimedia Magazine, vol. 9, no. 3 , pp. $42-55$, July 2002. 